\title{
AVALIAÇÃO DA TOXICIDADE AGUDA DO ANTIBIÓTICO CIPROFLOXACINA AOS CLADÓCEROS DAPHNIA MAGNA E CERIODAPHNIA SILVESTRII
}

Adrislaine da Silva Mansano $^{1}$

Laira Lúcia Damasceno de Oliveira²

Odete Rocha ${ }^{3}$

RESUMO: Nas últimas décadas a contaminação de origem antrópica dos ecossistemas aquáticos tem aumentado sucessivamente, e, em conseqüência, fármacos e seus resíduos são freqüentemente detectados nos ambientes aquáticos. A concentração crescente destes resíduos em ecossistemas aquáticos tem despertado cada vez mais interesse, tanto pelo aumento em quantidade e variedade, quanto pela toxicidade que pode provocar nos organismos aquáticos não-alvo, gerando assim a necessidade da avaliação dos seus efeitos nestes ecossistemas. Portanto, em virtude da existência de poucos estudos ecotoxicológicos com ciprofloxacina, o presente estudo teve como objetivo avaliar e comparar a toxicidade aguda deste antibiótico sobre as espécies de Cladocera Daphnia magna e Ceriodaphnia silvestrii. Para isso, realizaram-se testes de toxicidade aguda, com concentrações nominais do fármaco. Para o dafinídeo $C$. silvestrii, os valores de $\mathrm{CE}_{50} ; 24 \mathrm{~h}$ e $\mathrm{CE}_{50} ; 48 \mathrm{~h}$ foram de $43,80 \mathrm{mg} \mathrm{L}^{-1}$ e 9,17 $\mathrm{mg} \mathrm{L}^{-1}$, respectivamente. Para D. magna, os valores de $\mathrm{CE}_{50} 24$ e $48 \mathrm{~h}$ não foram possíveis de calcular, pois um número menor que $50 \%$ dos organismos apresentaram imobilidade na maior concentração testada (100 mg L $\left.{ }^{-1}\right)$. Os resultados obtidos mostraram que a espécie tropical $C$. silvestrii foi mais sensível ao antibiótico ciprofloxacina do que a espécie exótica $D$. magna. Muitos critérios de qualidade de água em regiões tropicais são baseados em

\footnotetext{
Bióloga. Universidade Federal de São Carlos, São Carlos, SP, Brasil. E-mail: laine_mansano@yahoo.com.br.

${ }^{2}$ Bióloga. Universidade de São Paulo, São Carlos, SP, Brasil. E-mail: lairaoliver@yahoo.com.br.

3 Bióloga. Professora Titular da Universidade Federal de São Carlos, São Carlos, SP, Brasil. E-mail: doro@ufscar.br
} 
testes ecotoxicológicos com espécies não-nativas e isso pode levar a erros na definição dos níveis máximos permitidos de substâncias químicas em corpos d'água. Portanto, este estudo sugere o uso de espécies nativas em avaliações ecotoxicológicas com compostos farmacológicos.

Palavras-chave: Antibiótico. Microcrustáceos. Fármacos.

\section{INTRODUÇÃO}

Nos últimos anos o monitoramento de fármacos no ambiente vem ganhando grande interesse devido ao fato de muitas dessas substâncias serem freqüentemente encontradas em efluentes de Estações de Tratamento de Esgoto (ETEs), águas de abastecimento (ETAs) e em outras matrizes ambientais como solo e sedimento em concentrações da ordem de $\mu \mathrm{g} \mathrm{L}^{-1}$ e $\mathrm{ng} \mathrm{L}^{-1}$ (BILA; DEZOTTI, 2003).

Os fármacos são considerados contaminantes ambientais devido a estas moléculas serem biologicamente ativas. Além disso, a grande maioria destes compostos tem facilidade de penetração nas barreiras biológicas, exercem ação farmacológica mesmo em baixas quantidades, exercendo ações distintas de acordo com a espécie animal e respectivas vias metabólicas envolvidas (FENT; WESTON; CAMINADA, 2006). Os fármacos têm também baixa biodegrabilidade no ambiente e elevada capacidade de bioacumulação e persistência ambiental, podendo adquirir novas formas (metabólitos ou produtos de degradação), com novos modos de ação e novos órgãos alvo (FENT; WESTON; CAMINADA, 2006).

O aporte de substâncias farmacológicas no ambiente advém do uso intenso e extensivo no tratamento de doenças em seres humanos e animais; sendo excretados na forma não metabolizada ou como metabólito ativo e introduzidas, principalmente, a partir do lançamento via efluentes municipais nos corpos hídricos receptores (CHAPMAN, 2006).

Dentre os fármacos, o grupo dos antibióticos é considerado um dos mais importantes. Estes compostos são extensivamente utilizados na medicina humana e veterinária, e, em aqüicultura, com o propósito de prevenir ou tratar infecções microbiais, embora na pecuária sejam também usadas como promotoras de crescimento. Em particular, os antibióticos representam uma especial ameaça para a saúde ambiental, 
devido a sua baixa biodegradabilidade e toxicidade para bactérias, além do potencial para o desenvolvimento de espécies bacterianas resistentes (KÜMMERER, 2004).

Os efeitos colaterais que os antibióticos podem causar, na maioria dos casos ainda são desconhecidos. De acordo com Jorgensen e Halling-Sorensen (2000), alguns desses efeitos podem ser observados em concentrações na ordem de $n g \mathrm{~L}^{-1}$ e já há indícios de que o desenvolvimento de resistência antibiótica seja ainda favorecido por essas baixas concentrações (SANTOS; PIZZOLATO; CUNHA, 2007).

Dentre as classes de antibióticos de maior relevância ambiental estão as fluoroquinolonas, cujo efeito antibacteriano baseia-se na inibição da enzima DNA-girase (topoisomerase II), envolvida na biossíntese de DNA (KATZUNG, 1995). A ciprofloxacina [1-cyclopropyl-6-fluoro-1,4-dihydro-4-oxo-7-(1-piperaziny)-3-quinoline carboxylic acid] (Figura 1) pertence ao grupo das fluoroquinolonas e atua contra uma ampla gama de bactérias Gram-negativas, incluindo várias espécies de Enterobacteriaceae e Pseudomonas, e bactérias Gram-positivas (CHIN; NEU, 1984). Comparado à fluoroquinolonas similares, como norfloxacina, ofloxacina, pefloxacina e enoxacina, a ciprofloxacina é aproximadamente 4 vezes mais ativa contra quase todas as bactérias citadas acima (VAN CAEKENBERGHE; PATTYN, 1984). Quando administrada oralmente, a ciprofloxacina é bem absorvida e distribuída nos tecidos e excretada na urina, em altas concentrações (81\% do administrado a humanos) (BORNER; LODE; HÖFFKEN, 1986).

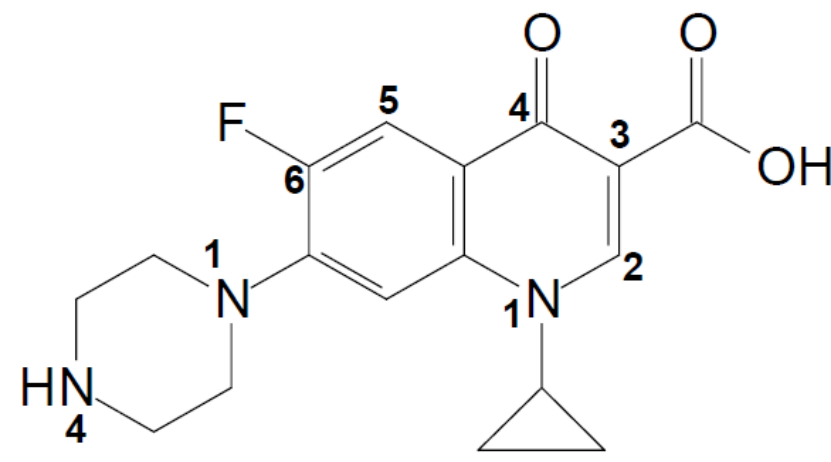

Figura 1: Estrutura da ciprofloxacina 
Segundo estudos, devido a sua ampla utilização, a ciprofloxacina é um contaminante freqüentemente encontrado no compartimento aquático. Este antibiótico foi verificado em efluentes hospitalares com valores entre 2 e $11 \mu \mathrm{g} \mathrm{L}^{-1}$ (BROWN et al. 2006; SEIFRTOVÁ et al. 2008), em efluentes de estações de tratamento de esgoto ( $<6$ - $310 \mathrm{ng}$ $\mathrm{L}^{-1}$ ) (BROWN et al. 2006; SEIFRTOVÁ et al. 2008), bem como em águas superficiais, como por exemplo no Rio Lambro (Itália) $\left(14,36 \mathrm{ng} \mathrm{L}^{-1}\right.$ ) (CALAMARI et al., 2003) e no Rio Mondego (Portugal) $\left(79,6\right.$ - 119,2 ng L ${ }^{-1}$ ) (PENA et al., 2007). No entanto, apesar de detectada em amostras de água naturais, os efeitos ecotoxicológicos da ciprofloxacina a organismos não-alvo, não foram amplamente investigados (SANTOS et al., 2010). Trabalhos anteriores demonstraram que a ciprofloxacina é extremamente genotóxico (HARTMANN et al., 1998) e apresenta toxicidade aguda a espécie de alga Pseudokirchneriella subcapitata (GRUNG et al., 2008). Alguns estudos foram realizados com a espécie Daphnia magna (e.g. HALLING-SORENSEN et al. 2000; MARTINS et al., 2012), no entanto, ainda não existem trabalhos de toxicidade aguda com ciprofloxacina utilizando a espécie de Cladocera Ceriodaphnia silvestrii.

Dentro deste contexto, o objetivo deste estudo foi avaliar e comparar a toxicidade aguda do antibiótico ciprofloxacina ao cladócero exótico Daphnia magna e ao cladócero nativo Ceriodaphnia silvestrii.

\section{MATERIAL E MÉTODOS}

\subsection{Organismos-teste}

A espécie Daphnia magna (Crustacea, Cladocera) é um microcrustáceo planctônico exótico, de larga distribuição no hemisfério norte e com 5 a $6 \mathrm{~mm}$ de comprimento. A espécie Ceriodaphnia silvestrii (Crustacea, Cladocera) é um microcrustáceo planctônico nativo, encontrado no Brasil e na Argentina e que apresenta de 0,8 a 0,9 mm de comprimento. Em geral os cladóceros da família Daphnidae são os organismos-teste preferencialmente utilizados em ensaios toxicológicos devido à facilidade de cultivo, ao pequeno porte, à representatividade na comunidade zooplanctônica e por terem ciclo de vida relativamente curto (RAND, 1995). 


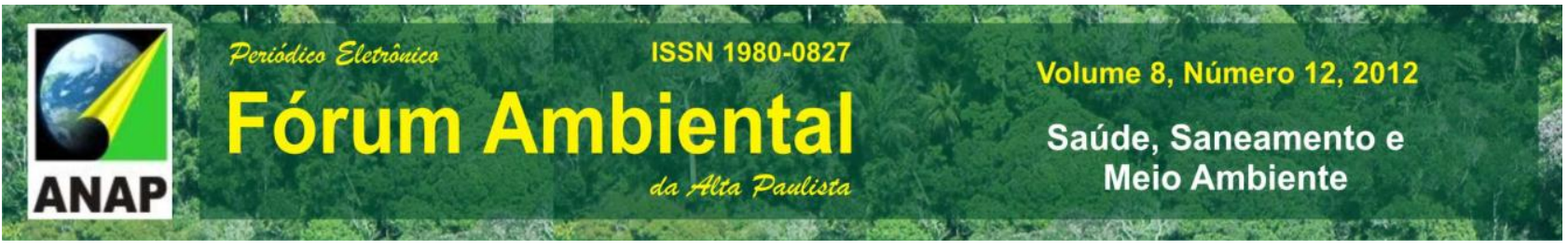

A espécie D. magna é um organismo amplamente utilizado em Ecotoxicologia na avaliação da toxicidade de várias substâncias químicas. No entanto, como este organismo não ocorre naturalmente em corpos d'água brasileiros, a utilização da espécie nativa $C$. silvestrii neste estudo para avaliação da toxicidade aguda da ciprofloxacina tem o intuito de possibilitar maior representatividade quando se extrapolar os dados de ensaios em laboratório às condições de campo, aumentando a eficiência e a confiabilidade das avaliações ecotoxicológicas.

Os indivíduos de $C$. silvestrii e $D$. magna utilizados nos bioensaios ecotoxicológicos foram cultivados no laboratório de Ecotoxicologia do Departamento de Ecologia e Biologia Evolutiva da Universidade Federal de São Carlos (DEBE/UFScar).

\subsection{Manutenção e cultivo das espécies de Cladocera}

Os cultivos de $D$. magna foram mantidos em incubadora com temperatura controlada a $22 \pm 2^{\circ} \mathrm{C}$, enquanto os de $C$. silvestrii foram mantidos a $25 \pm 2^{\circ} \mathrm{C}$, ambas com fotoperíodo de $12 \mathrm{~h}$ (claro)/12h(escuro). Para o cultivo da $C$. silvestrii foi utilizada água reconstituída preparada de acordo com a ABNT (2005) e com as seguintes características: $\mathrm{pH}$ de 7,0 a 7,6, dureza entre 40 e $48 \mathrm{mgCaCO}_{3} \mathrm{~L}^{-1}$ e condutividade elétrica de $160 \mu \mathrm{S} \mathrm{cm}^{-1}$. Essa espécie de cladócero foi mantida em béqueres de $2 \mathrm{~L}$, com um máximo de 140 indivíduos por recipiente.

A espécie $D$. magna foi mantida em meio de cultura artificial de elevada dureza (ASTM, 1980), com as seguintes características: pH 8,0 a 8,6 e dureza entre 180 e 220 $\mathrm{mgCaCO}_{3} \mathrm{~L}^{-1}$. Essa espécie foi mantida em béqueres de $2 \mathrm{~L}$, com um máximo de 50 indivíduos por recipiente. Os indivíduos de C. silvestrii foram alimentados com suspensão algacéa de Pseudokirchneriella subcapitata, cultivada em meio CHU-12 (MÜLLER, 1972), a concentração de $1 \times 10^{5}$ células $\mathrm{mL}^{-1}$ e alimento composto (levedura e ração de peixe fermentada na proporção de 1:1) numa concentração de $1 \mathrm{~mL} \mathrm{~L}^{-1}$ (ABNT, 2004). $A$ espécie $D$. magna foi alimentada conforme descrito para a $C$. silvestrii, porém com 0 dobro de suspensão algacéa $\left(2 \times 10^{5}\right.$ células $\left.\mathrm{mL}^{-1}\right)$.

A manutenção das culturas foi realizada três vezes por semana, quando eram renovados a água e o alimento. Mensalmente, a saúde dos cultivos foi verificada por 
testes de toxicidade aguda com a substância de referência cloreto de sódio para a espécie $C$. silvestrii e dicromato de potássio para a $D$. magna.

\subsection{Testes de toxicidade aguda}

O antibiótico ciprofloxacina utilizado possui grau de $90 \%$ de pureza, Marca Eurofarma. A solução estoque foi preparada através da diluição de ciprofloxacina em água destilada, numa concentração de $500 \mathrm{mg} \mathrm{L}^{-1}$. Os procedimentos metodológicos foram realizados de acordo com norma padronizada (ABNT, 2004). Os testes de toxicidade aguda consistiram na exposição de 5 neonatas com menos de 24 horas de idade por réplica, à diferentes concentrações de ciprofloxacina $(3,125 ; 6,25 ; 12,5 ; 25 ; 50$ e $100 \mathrm{mg} \mathrm{L}^{-1}$ ) estabelecidas de acordo com Martins et al. (2012) para D. magna. Para a $C$. silvestrii utilizou-se as mesmas concentrações para possível comparação entre as duas espécies estudadas. Os testes de toxicidade tiveram a duração de 24 e 48 horas e foram realizados em placas de cultivo celular de 6 poços $\left(\right.$ TPP $\left.^{\circledR}\right)$. Como controle utilizou-se água reconstituída. Foram realizadas quatro repetições tanto para o controle como para cada concentração de ciprofloxacina testada, sendo que em cada réplica foram adicionados 10 $\mathrm{mL}$ de solução-teste. Os experimentos foram mantidos sob a mesma temperatura dos cultivos-estoque, sem iluminação e alimentação. No início dos testes foram realizadas medidas das variáveis $\mathrm{pH}$, condutividade, temperatura e dureza, sendo que todas estas variáveis foram medidas também no final do teste, com exceção da dureza que apresentou volume insuficiente.

Após o período de exposição (24 e 48 horas) procedeu-se à contagem dos organismos imóveis. Se a porcentagem de indivíduos imóveis no controle excedesse $10 \%$, o teste era considerado inválido, o que não ocorreu nesse experimento. Os resultados foram expressos em $\mathrm{CE}_{50}$ - concentração efetiva mediana que causa efeito agudo a 50\% dos organismos no tempo de exposição (ABNT, 2004), com intervalo de confiança de 95\%, utilizando-se o programa estatístico Trimmed Spearmann- Karber (HAMILTON; RUSSO; THURFTON, 1977).

\section{RESULTADOS E DISCUSSÃo}


Durante os testes de toxicidade aguda para a Ceriodaphnia silvestrii, os valores do $\mathrm{pH}$ das soluções testes permaneceram dentro da faixa de 7,0 a 7,6 e não variaram mais do que 0,5 unidade durante o teste. A temperatura variou de 24,3 a 24,6 ${ }^{\circ} \mathrm{C}$, a condutividade elétrica variou de 131,7 a $157,7 \mu \mathrm{S} \mathrm{cm}^{-1}$ e a dureza inicial variou de 40 a 48 mg $\mathrm{CaCO}_{3} \mathrm{~L}^{-1}$. Durante os testes de toxicidade aguda para a Daphnia magna, os valores do $\mathrm{pH}$ das soluções testes mantiveram dentro da faixa de 8,0 a 8,6 e não variaram mais do que 0,5 unidade durante o teste. A temperatura variou de 24,4 a $24,6{ }^{\circ} \mathrm{C}$, a condutividade elétrica variou de 449 a $542 \mu \mathrm{S} \mathrm{cm}^{-1}$ e a dureza inicial variou de 180 a 184 $\mathrm{mg} \mathrm{CaCO}_{3} \mathrm{~L}^{-1}$. Assim, os testes de toxicidade preencheram os critérios de validade estabelecidos nas diretrizes da Associação Brasileira de Normas Técnicas (ABNT, 2005) para C. silvestrii e da "Organization for Economic Cooperation and Development" (OECD, 2004) para D. magna.

A Figura 2 apresenta o número de organismos imóveis no controle e nas concentrações testadas para C. silvestrii e D. magna expostos ao antibiótico ciprofloxacina durante 48 horas. Pela análise da Figura 2, observou-se que, enquanto na maior concentração testada $\left(100 \mathrm{mg} \mathrm{L}^{-1}\right), 100 \%$ dos organismos de $C$. silvestrii apresentaram imobilidade, para D. magna, apenas $30 \%$ dos organismos apresentaram imobilidade. Os valores da $\mathrm{CE}_{50} ; 24 \mathrm{~h}$ e $\mathrm{CE}_{50}$; $48 \mathrm{~h}$ e os intervalos de confiança obtidos nos testes realizados com os dois cladóceros estão apresentados na Tabela 1. Como menos de $50 \%$ dos organismos de D. magna apresentaram imobilidade, não foi possível calcular o valor de $\mathrm{CE}_{50}$ para esta espécie.
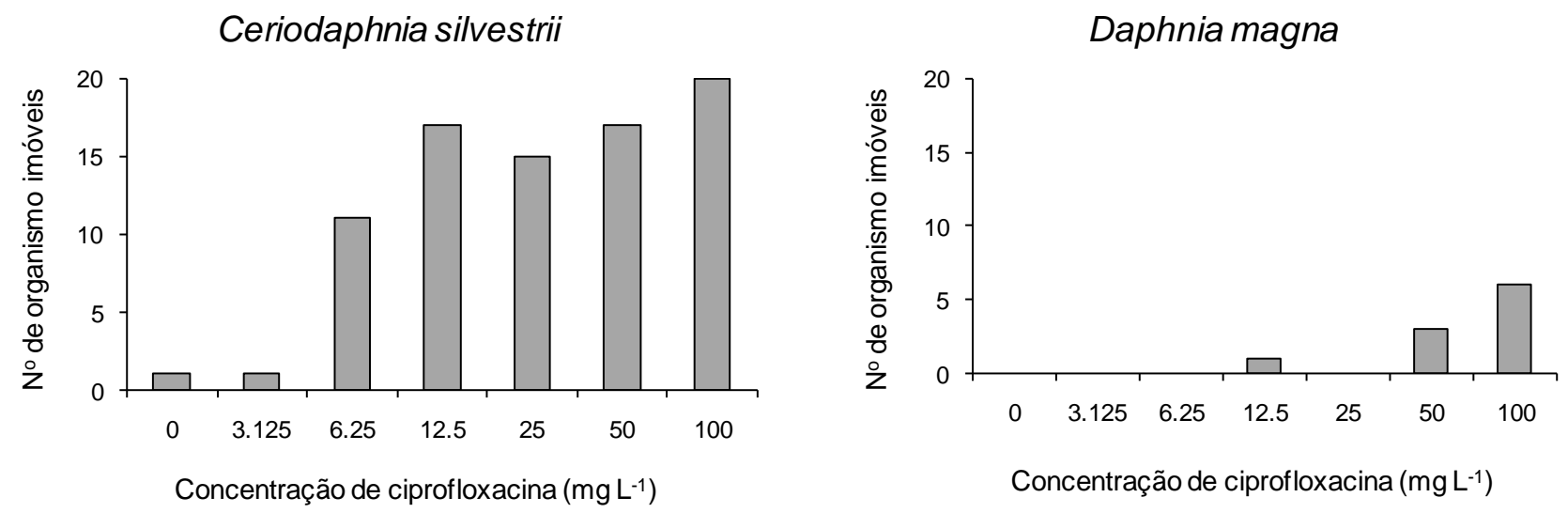
Figura 2: Número de organismos imóveis no controle e nas concentrações testadas para $C$. silvestrii e $D$. magna expostos ao antibiótico ciprofloxacina durante 48 horas

Nota: O número de organismos imóveis refere-se à somatória das quatro réplicas

Tabela 1: Valores de $\mathrm{CE}_{50} 24$ e 48 horas do antibiótico ciprofloxacina e respectivos intervalos de confiança (IC- 95\%), para os cladóceros Ceriodaphnia silvestrii e Daphnia magna, em testes de toxicidade aguda

\begin{tabular}{cccc}
\hline Organismo-teste & Tempo de exposição (h) & $\mathbf{C E}_{50}\left(\mathbf{m g ~ L}^{-1}\right)$ & Intervalo de Confiança (IC-95\%) \\
\hline \multirow{2}{*}{ Ceriodaphnia silvestrii } & 24 & 43,80 & $31,23-61,44$ \\
& 48 & 9,17 & $7,05-11,91$ \\
\multirow{2}{*}{ Daphnia magna } & 24 & NC & NC \\
& 48 & NC & NC \\
\hline
\end{tabular}

Nota: NC= Não foi possível calcular

De acordo com os resultados, observou-se que a espécie nativa Ceriodaphnia silvestrii foi mais sensível a ciprofloxacina do que a espécie exótica Daphnia magna, tanto em 24 horas quanto em 48 horas de exposição ao antibiótico.

Martins et al. (2012) determinaram a $\mathrm{CE}_{50}$ para Daphnia magna exposta a ciprofloxacina, e observaram que esta foi de $65,3 \mathrm{mg} \mathrm{L}^{-1}$. Halling-Sorensen et al. (2000) não observaram, como no presente estudo, a $C_{50}$ para $D$. magna, devido ao fato da concentração máxima testada $\left(60 \mathrm{mg} \mathrm{L}^{-1}\right)$ não causar efeito agudo a $50 \%$ dos organismos.

Para a espécie C. silvestrii, não foram encontrados na literarura estudos ecotoxicológicos ao antibiótico ciprofloxacina. Na Tabela 2 são apresentados dados ecotoxicológicos $\left(\mathrm{EC}_{50} ; \mathrm{mg} \mathrm{L}^{-1}\right)$ para diferentes organismos aquáticos expostos a ciprofloxacina disponíveis na literatura. Ao comparar a $\mathrm{CE}_{50}$ observada para a $C$. silvestrii a de outras espécies aquáticas, percebeu-se que esta foi mais sensível a ciprofloxacina do que as espécies Vibrio fischeri, Chlorella vulgaris, Daphnia magna, Gambusia holbrooki e Danio rerio (Tabela 2). No entanto, diferentes causas da variabilidade na sensibilidade de espécies para um determinado poluente incluem características fisiológicas dos animais selecionados (estado de nutrição, sexo, estágio no ciclo de vida), as condições de cultura (temperatura, alimentação, fotoperíodo e dureza da água) e também propriedades das substâncias utilizadas nos ensaios (degradabilidade, solubilidade e pureza) (COWGILL, 1987; RAND, 1995). 
Tabela 2: Valores de $\mathrm{CE}_{50}\left(\mathrm{mg} \mathrm{L}^{-1}\right)$ para diferentes organismos aquáticos expostos ao antibiótico ciprofloxacina disponíveis na literatura

\begin{tabular}{lccc}
\hline \multicolumn{1}{c}{ Organismo-teste } & Parâmetro & CE $_{50}$ ( $\mathbf{m g ~ L}^{-1}$ ) & Referência \\
\hline Vibrio fischeri & Inibição de luminescência & $>5,9$ & Hernando et al. (2007) \\
& Inibição de luminescência & 11,5 & Martins et al. (2012) \\
Pseudokirchneriella subcapitata & Inibição de crescimento - 72h & 2,97 & Halling-Sorensen et al. (2000) \\
& Inibição de crescimento - 96h & 4,83 & Martins et al. (2012) \\
Chlorella vulgaris & Inibição de crescimento - 96h & 20,6 & Ni et al. (2008) \\
Microcystis aeruginosa & Inibição de crescimento - 120h & 0,017 & Robinson et al. (2005) \\
Lemna gibba & Inibição de crescimento - 7 dias & 0,697 & Brain et al. (2004) \\
Lemna minor & Inibição de crescimento - 7 dias & 3,75 & Martins et al. (2012) \\
Daphnia magna & Imobilização - 48h & $>60$ & Halling-Sorensen et al. (2000) \\
& Imobilização - 48h & $>10$ & Robinson et al. (2005) \\
Gambusia holbrooki & Imobilização - 48h & 65,3 & Martins et al. (2012) \\
Danio rerio & Mortalidade - 96h & $>60$ & Martins et al. (2012) \\
\hline
\end{tabular}

O uso de espécies de clima temperado em regiões tropicais para as avaliações ecotoxicológicas parece ser inadequado, uma vez que as respostas tóxicas de organismos a substâncias químicas são freqüentemente espécie-específicas. Portanto, o uso destas espécies nos trópicos tem sido questionado (DO HONG et al., 2004).

Muitos critérios de qualidade de água em regiões tropicais são baseados em testes ecotoxicológicos com espécies não-nativas e, conseqüentemente, isso pode levar a erros na definição dos níveis máximos permitidos de substâncias químicas em corpos d'água. Portanto, este estudo reforça-se a idéia de utilizar espécies nativas para estabelecer as concentrações máximas de substâncias tóxicas em critérios de qualidade da água, uma vez que os seus efeitos podem estar relacionados com variáveis ambientais específicas para cada região.

Embora as concentrações de efeito obtidas para a espécie Ceriodaphnia silvestrii estejam muito acima dos níveis de ciprofloxacina quantificados nos sistemas aquáticos (e.g. PENA et al. 2007; SEIFRTOVÁ et al. 2008), a capacidade deste antibiótico de persistir adsorvido em partículas, em conjunto com a entrada contínua no ambiente (ROBINSON et al., 2005) pode constituir um risco para a biota aquática. Ensaios crônicos realizados por Martins et al. (2012) com D. magna evidenciaram que uma exposição 
prolongada a baixas concentrações de ciprofloxacina induziu deficiências aos parâmetros de ciclo de vida desta espécie (tamanho dos neonatos, fecundidade, número de ninhadas por fêmea, dentre outros). Tal resultado pode antecipar potenciais danos sobre a manutenção a longo prazo de populações naturais expostas continuamente a entrada de antibióticos, além de reforçar a necessidade de testes de toxicidade crônica paralelamente aos testes agudos.

\section{CONCLUSÕES}

De acordo com os resultados de toxicidade e comparações entre os cladóceros estudados, concluiu-se que a espécie nativa Ceriodaphnia silvestrii foi mais sensível ao antibiótico ciprofloxacina do que a espécie exótica Daphnia magna. Assim, o uso de espécies de clima temperado em regiões tropicais para as avaliações ecotoxicológicas deve ser questionado, propondo-se o uso de espécies nativas que podem fornecer mais informações ecologicamente relevantes sobre a toxicidade dos fármacos nos ambientes aquáticos tropicais. No entanto, mais estudos de toxicidade aguda e crônica devem ser realizados para a avaliação dos diferentes efeitos (mortalidade, reprodução, desenvolvimento tardio e malformações) deste composto nos ecossistemas aquáticos, a fim de gerar dados para integrar a avaliação de risco de fármacos e proteger os organismos não-alvo da ameaça representada pela presença destes produtos no ambiente.

\section{REFERÊNCIAS}

ABNT - Associação Brasileira de Normas Técnicas NBR 12713. Ecotoxicologia aquática Toxicidade Aguda - Método de ensaio com Daphnia spp. (Cladocera, Crustacea). Rio de Janeiro, 21p, 2004.

ABNT - Associação Brasileira de Normas Técnicas NBR 13373. Ecotoxicologia aquática Toxicidade crônica - Método de ensaio com Ceriodaphnia spp. (Crustacea, Cladocera). Rio de Janeiro, 15p, 2005. toxicity test with fishes, macroinvertebrates and amphibians. E-790-80. Philadelphia, 1980. 
BILA, D.M.; DEZOTTI, M. Fármacos no meio ambiente. Química Nova, v. 26, n. 4, p. 523-530, 2003.

BORNER, K., LODE, H. \& HÖFFKEN, G. Renal elimination of sulfociprofloxacin, a new metabolite of ciprofloxacin. European Journal of Clinical Microbiology, v. 5, p. 476, 1986.

BRAIN, R.A.; JOHNSON, D.J.; RICHARDS, S.M.; SANDERSON, H.; SIBLEY, P.K.; SOLOMON, K.R. Effects of 25 pharmaceutical compounds to Lemna minor using a seven-day static renewal test. Environmental Toxicology and Chemistry, v. 2, p. 371-382, 2004.

BROWN, K.D.; KULIS, J.; THOMSON, B.; CHAPMAN, T.H.; MAWHINNEY, D.B. Occurrence of antibiotics in hospital, residential, and dairy effluent, municipal wastewater, and the Rio Grande in New Mexico. Science of the Total Environment, v. 366, p. 772-783, 2006.

CALAMARI, D.; ZUCCATO, E.; CASTIGLIONI, S.; BAGNATI, R.; FANELLI, R. Strategic survey of therapeutic drugs in the Rivers Po and Lambro in Northern Italy. Environmental Science and Technology, v. 37, p. 1241-1248, 2003.

CHAPMAN, P. M. Emergin substances - Emerging problems? Environmental Toxicology and Chemistry, v. 25, n. 6, p. 1445-1447, 2006.

CHIN, N.; NEU, H.C. Ciprofloxacin, a quinoline carboxylic acid compound active against aerobic and anaerobic bacteria. Antimicrob Agents Chemother, v. 25, p. 319-326, 1984.

COWGILL, U.M. Critical analysis of factors affecting the sensitivity of zooplankton and the reproducibility of toxicity test results. Water Research, v. 21, p. 1453-1462, 1987.

DO HONG, L.C.; BECKER-VAN SLOOTEN, K.; TARRADELLAS, J. Tropical ecotoxicity testing with Ceriodaphnia cornuta. Environmental Toxicology, v. 1, p. 497-504, 2004.

FENT, K.; WESTON, A.; CAMINADA, D. Ecotoxicology of human pharmaceuticals, Aquatic Toxicology, v. 76, n. 9, p. 122-159, 2006.

GRUNG, M.; KALLQVIST, T.; SAKSHAUG, S.; SKURTVEIT, S.; THOMAS, K.V. Environmental assessmentof Norwegian priority pharmaceuticals based on the EMEA guideline. Ecotoxicology and Environmental Safety, v. 71, p. 328-340, 2008.

HALLING-SORENSEN, B.; LUTZHOFT, H.; ANDERSEN, H.; INGERSLEV,F. Environmental risk assessment of antibiotics: comparison of mecillinam, trimethoprim and ciprofloxacin. Journal of Antimicrobial Chemotherapy, v. 46, p. 53-58, 2000. 
HAMILTON, M.A.; RUSSO, R.C.; THURFTON, R.B.Trimmed Spearman-Karber method for estimating median lethal concentration in toxicity bioassays. Environmental Science and Technology, v. 11, n. 7, p. 714-719, 1977.

HARTMANN, A.; ALDER, A.C.; KOLLER, T.; WIDMER, R.M. Identification of fluoroquinolone antibiotics as the main source of $u m u C$ genotoxicity in native hospital wastewater. Environmental Toxicology and Chemistry, v. 17, n. 3, p. 377-382, 1998.

HERNANDO, M.D.; DE VETTORI, S.; MARTÍNEZ BUENO, M.J.; FERNÁNDEZ-ALBA, A. Toxicity evaluation with Vibrio fischeri test of organic chemicals used in aquaculture. Chemosphere, v. 68, p. 724-730, 2007.

JORGENSEN, S. E.; HALLING-SORENSEN, B. Drugs in the enviroment. Chemosphere, v.40, no7, p.691-699, 2000.

KATZUNG, B.G. Farmacologia básica e clínica. $6^{\circ}$ ed. Rio de Janeiro: Guanabara Koogan, 1995. $516 \mathrm{p}$.

KÜMMERER, K. Pharmaceuticals in the environment: sources, fate, effects and Risks, $2^{a}$ ed., Berlin: Springer-Verlag, 2004. 240 p.

MARTINS, N.; PEREIRA, R.; ABRANTES, N.; PEREIRA, J.; GONÇALVES, F.; MARQUES, C.R. Ecotoxicological effects of ciprofloxacin on fresh water species: data integration and derivation of toxicity thresholds for risk assessment. Ecotoxicology, v. 21, p. 1167-1176, 2012.

MÜLLER, H. Wachstum and phosphatbedarf von Nitzschia actinastroides (Lemn.) v. Goor in statischer und homokontiuierliecher kultur unter phosphatlimitierung. Archiv fuer Hydrobiologie Supplement, v. 38, p. 399-484, 1972.

NI, X.P.; WANG, X.; CHEN, J.; ZITKO, V.; NA,T. Response of the freshwater Chlorella vulgaris to trichloroisocyanuric acid and ciprofloxacin. Environmental Toxicology and Chemistry, v. 27, n. 1, p. 168-173, 2008.

OECD - Organization for Economic Cooperation and Development. Daphnia sp., acute immobilization test. Guidelines for testing of chemicals, $\mathbf{n}^{\circ} .202$. Organization for Economic Cooperation and Development, Paris, 2004.

PENA, A.; CHMIELOVA, D.; LINO, C.M.; SOLICH, P. Determination of fluoroquinolone antibiotics in surface waters from Mondego River by high performance liquid chromatography using amonolithic column. Journal of Separation Science, v. 30, p. 2924-2928, 2007. 
RAND, G.M. Fundamental Aquatic Toxicology: Effects, Environmental Fate, and Risk Assessment, $2^{\mathrm{a}}$ ed. Taylor \& Francis: USA, cap 31, 1995.

ROBINSON, A.A.; BELDEN, J.B.; LYDY, M.J. Toxicity of fluoroquinolone antibiotics to aquatic organisms. Environmental Toxicology and Chemistry, v. 24, p. 423-430, 2005.

SANTOS, J.H.Z.; PIZZOLATO, T.M.; CUNHA, A.C.B. Desenvolvimento de metodologia analítica para quantificação de fármacos em meio aquático por extração em fase sólida e HPLC. Revistas de ciências ambientais, v.1, n.2, p. 19-34, 2007.

SANTOS, L.H.M.L.M.; ARAÚJO, A.N.; FACHINI, A., PENA, A.; DELERUE-MATOS, C.; MONTENEGRO, M.C. Review - ecotoxicological aspects related to the presence of pharmaceuticals in the aquatic environment. Journal of Hazardous Materials, v. 175, p. 45-95, 2010.

SEIFRTOVÁ, M.; PENA, A.; LINO, C.M.; SOLICH, P. Determination of fluoroquinolone antibiotics in hospital and municipal wastewaters in Coimbra by liquid chromatography with a monolithic column and fluorescence detection. Analytical and Bioanalytical Chemistry, v. 391, p. 799-805, 2008.

VAN CAEKENBERGHE, D.L.; PATTYN, S.R. In vitro activity of ciprofloxacin compared with those of other new fluorinated piperazinyl-substituted quinoline derivatives. Antimicrob Agents

Chemother, v.25, p. 518-521, 1984. 